\title{
Improve the Performance of Orthogonal Frequency Division Multiplexing Based Discrete Wavelet Transform Using Cooperative Communication System
}

\author{
Dr. Mohammed Aboud Kadhim \\ Middle Technical University, Institute of Technology Baghdad, Iraq
}

\begin{abstract}
Cooperative communication mean remote hubs can help each other for communication. In this work, contemplate agreeable "Symmetrical Recurrence Division Multiplexing (OFDM)" based "Discrete wavelet change (DWT)".OFDM Cooperative 1 plan for multi-hub remote systems utilizing both Open up and-Forward (AF) and Decipher and-Forward (DF) handing-off. In this paper, an enhance the execution OFDM-DWT. We expect that the source and the transfer are gathered. Subsequent to interpreting, the transfer rearranges the information before transmitting towards the goal. Thus, the goal gets two individual imitations of the transmit flag and utilize decent variety consolidating. The bit mistake execution is offered, for the assembled organize. BER reenactment in channels An and B demonstrates that the handing-off convention bolstered agreeable OFDM-DWT framework beats flag. Recreation results express that the recommended remapping assorted variety strategy at the hand-off accomplishes a superior execution of OFDM-DWT based arranged hand-off correspondences
\end{abstract}

Keywords-OFDM, DWT, AF, DF, cooperative

DOI: $10.7176 / \mathrm{NCS} / 10-03$

Publication date:July $31^{\text {st }} 2019$

\section{I.Introduction}

On this cutting-edge have a look at, Orthogonal frequency division multiplexing (OFDM) based totally cooperative protocol has been applied. In broadband wi-fi communications. OFDM is an energetic techniques to alleviate the inter-image interference (ISI) and provide high spectral performance. OFDM is utilized in several communications schemes like wi-fi neighborhood location networks (WLANs), wi-fi non-public vicinity networks (WPANs) and so forth. To mend the overall performance of OFDM schemes, the crucial concept of cooperative variety may be implemented [1]. As of overdue, multihop communication has end up right into a dynamic also, crucial scope of studies in wireless communication. In a cellular network, relaying schemes supply promising advances to transmit at better facts fee to dreams more remote away [2-6]. With a selected end aim to coverage vicinity, a couple of relays have to be set within the cell shape. Thus, some clients will truely be in the cowl of the scope locales of neighboring relays. On this way it's far charming to bear in mind how to transmit statistics to severa clients making use of multiple relays cooperatively. Concentrated on at increasing the channel capability and/or decreasing the outage probability, numerous noticeable cooperative protocols have been suggested [1]. Between, them, amplify-forward (AF) and decode-forward (DF) are the two important forwarding modes. Their characteristic have been considered by numerous scientists both from the information theoretic facet and the feasible facets $[2,3]$ estimated their performances in feasible wireless situations in common, and the inter-user outage situation in specific. The current work of[4] has suggested a novel hybrid forwarding system for OFDM-based cooperative relay networks. At the relay node level, this system adaptively selects to switch between AF and DF methods depending on the channel link kinds. In [5] an AF based relaying OFDM system has been presented to perform prospective generation $(4 \mathrm{G})$ mobile networks. In this system, the relay reorganizes OFDM subcarriers to get optimum operation. Relaying ideas have also been suggested to improve the performance of WiMAX schemes [6]. In this paper, we regard a collocated source-relay and destination communication scheme based on OFDM-DWT. Wavelet transformation has lately be disclosed as a powerful nominee for digital communications [7]. In DFT-OFDM schemes, signals only overlap in the frequency domain though DWT-OFDM signals overlap both in the time and frequency domains, so there is no essential for the CP as in the DFT-OFDM situation. So, by this transformation, the spectral inclusion of the channel is enhanced [8]. The relay is supposed to be in the proximity of the source, so the channel paths of source-destination and relaydestination are greatly correlated. Hence the attainable spatial diversity gain, using DF relaying becomes less than two. The overview of OFDM into relay communications, offers a unique suitable to profiteer space/frequency diversities. Hence when space diversity is scarce, performance developments be able to be made if we can activeness, on the frequency diversity given by OFDM transmissions. The paper is structured as follows: The scheme model is discussed in Section II. Simulation Results and discussion will be debated in Section III, conclusions will be debated in Section IV. 


\section{II.System Model.}

The OFDM-DWT relay communication scheme regarded in this paper. One source terminal, S, one destination terminal, $\mathrm{D}$, and a relay $\mathrm{R}$ helping the transmission of the source is regarded. The three terminals, $\mathrm{S}, \mathrm{R}$ and $\mathrm{D}$ are supplied with single antennas. At $\mathrm{S}$, the binary data bits are serial-to-parallel converted and mapped onto a signal constellation. Let the transmitted 16-QAM constellation point on the $k^{\text {th }}$ subcarrier. The discrete time domain OFDM signal samples got after performance an inverse discrete Wavelet transform (IDWT) to $X=$ $[X(0), X(1), \ldots, X(N-1)]$ is expected by $N$ is the total number of subcarriers of the OFDM-DWT scheme. s. At the relay/destination, samples $x(n), n=0 \ldots(N-1)$ are collected and a N-point DWT is applied to repossess the transmitted data on all subcarriers. In Wavelet based OFDM-DWT, the time-windowed complex exponentials are substitute by wavelet "carriers", at diverse scales (j) and locations on the time axis (k). These functions are produced by the translation and dilation of a unique function, named "wavelets mother" and represented by $\psi(\mathrm{t})$ :

$$
\Psi_{L, K}(t)=2^{\frac{-l}{2}} \Psi\left(2^{-l} t-k\right)
$$

The orthogonally of these carriers depend on time location $(\mathrm{k})$ and scale index $(l)$. Wavelet carriers declare enhanced time-frequency localization than complex exponentials though DWT-OFDM employment complexity is comparable to that of FFT- OFDM. The important point 'orthogonally' is attained by producing members of a wavelet family, according to equation (1)

$$
\Psi_{L, K}(t), \Psi_{m, n}(t)=\left\{\begin{array}{l}
1, l=m \text { and } k=n \\
0 \text { Otherwise }
\end{array}\right.
$$

These functions have orthonormal basis, if infinite number of scales $l \in Z_{l}$ are measured. To get finite number of scales, scaling function $\Phi(t)$ is used. DWT-OFDM symbol now be able to be measured as weighted sum of wavelet and scale carriers, as declared in equation (3). This is near to the Inverse Wavelet Transform (IDWT).

$$
X(t)=\sum_{l \leq L} \sum_{k} w_{l, k}(t) \Psi_{L, K}(t)+\sum_{k} a_{L, k} . \Phi_{L, k}(t)
$$

$X$ The data symbols are understood by IDWT modulator as sequence of wavelet $w_{l, k}$, and estimate coefficients $a_{L, k}$.Agreeing to Equation (1) $L$ is the scale with poorest time resolution and good frequency localization of the carriers. For calculating IDWT, Mallet's procedure based on filter bank is used in its place of Equation (3). At the output of the filter discrete form of DWT-OFDM symbol is got, with impulse response of filters (low-pass and high-pass) definite by the mother wavelet. The transmitted signals engender through a multipath wireless channel which is demonstrated in the time domain by L delayed impulses

$$
h(t, \tau)=\sum_{l=0}^{L-1} h(l) \delta\left(t-\tau_{l}\right)
$$

At which $\delta(\cdot)$ is the Dirac function and $h(l)$ s are zero-mean complex Gaussian random mutable OFDM schemes are intended with $L_{C}<\mathrm{N}$. So in multipath fading, frequency domain channel constants $H(k)$ are dissimilar then correlated. The correlation coefficient, $\rho \Delta$ rely on the divergence between $k t h$ and $l$ th frequency bins. For kth and lth bins that are necessarily parted in frequency, the channel correlation is insignificant. The correlation constant is mathematically assumed by

$$
\rho \Delta=E\left[H(k) H^{*}(k+\Delta)\right]=E\left[\sum_{l=0}^{L-1} h(l) e^{\frac{-j 2 \pi k l}{N}} \sum_{s=0}^{L-1} h^{*}(s) e^{\frac{-j 2 \pi(k+\Delta k) s}{N}}\right]
$$

Wherever (.) $)^{*}$ is the complex union and E [.] is the expectation operator. Equation. (4) For this channel be able to be easy using the characteristics of the geometric series and the correlation constant is assumed by 
$\rho \Delta=\frac{1}{L} \frac{\sin \left(\frac{\pi \Delta K L}{N}\right)}{\sin \left(\frac{\pi \Delta K}{N}\right)} e^{j \pi \Delta k(L-1) / N}$

In AAF relaying procedure, the signal received by the relay is reduced and it is wanted to be amplified before it is directed up again. In responsibility thus, the noise in the signal is amplified also, this is the chief disadvantage of this protocol. The received signal is amplified block wise. Supposing that the channel typical is estimated completely, the gain for the amplification be able to be considered as follows. The power of the received OFDMDWT signal is assumed by:

$$
E\left|Y_{r}\right|^{2}=E\left|H_{s, r}\right|^{2} * E\left|X_{s}\right|^{2}+E\left|z_{s, r}\right|^{2}=E\left|H_{s, r}\right|^{2} \xi+2 \sigma_{s, r}^{2}
$$

Wherever s means the source, $\mathrm{r}$ the relay and $\xi=E\left|X_{S}\right|^{2}$ means the energy of the transmitted signal. To direct the data with the similar power as the transmitter did, the relay has to usage a gain any be able to be expressive:

$$
\beta=\sqrt{\frac{\xi}{\left|H_{s, r}\right|^{2} \xi+2 \sigma_{s, r}^{2}}}
$$

This word has to be computed for each block and so the channel specific of each single block necessities to be valued. In DAF relaying protocol, the source node transmits its own particular data both to the goal node and the transfer node in the meantime. The transfer node translates it, and forward to the goal node. The upside of interpret and-forward is that if the data contains errors, the mistake can be remedied in the relay and the mistake message couldn't be sent to the goal node. The got flag's quality can be significantly moved forward. Nonetheless, in the DAF relaying protocol, as the transfer node needs to interpret the got motion from source node and forward to the goal node, it will build the multifaceted nature of relay and cost. When using signal combining techniques, In Equal Ratio Combining (ERC), the received signals are only addend up before the symbols are discovered. It is to be eminent here that we necessity to identify the information of the phase shift of the signal to happen caused by multipath fading. The expected signal in ERC is assumed by

$$
Y_{d}[n]=Y_{s, d}[n] e^{-\angle H_{s, d}[n]}+Y_{r, d}[n] e^{-\angle H_{r, d[n]}}
$$

The coefficients $Y_{s, d}[n], d$ and $Y_{r, d}[n]$ mean the received signal from the transmitter and the relay. In Fixed Ratio Combining, the received signals are weighted with a constant ratio. Its ideal value is approached by entrustment dissimilar values in simulation to obtain a thought, on its suitable, chosen value. The expected signal in FRC is assumed by

$$
Y_{d}[n]=d_{s, d} Y_{s, d}[n] e^{-\angle H_{s, d}[n]}+d_{r, d} Y_{r, d}[n] e^{-\angle H_{r, d}[n]}
$$

In Signal to Noise Ratio Combining (SNRC), a considerable improved performance be able to be attained when the present state of dissimilar channel is identified exactly. A frequently used value to describe the magnitude value of a link is the SNR which is used to weight the incoming signals. The incoming signal in SNRC is assumed by:

$$
Y_{d}[n]=S N R_{s, d} Y_{s, d}[n] e^{-\angle H_{s, d}[n]}+S N R_{r, d} Y_{r, d}[n] e^{-\angle H_{r, d[n]}}
$$

In Enhanced Signal to Noise Ratio Combining (ESNRC), a received signal is ignored in situation of other received signal have a much improved value. If the channels have additional or less the similar channel value, the received signals are codified evenly. We be able to reform the SNR value of the channel for which this sentence be able to be made. In this simulation study, a SNR value of $10 \mathrm{~dB}$ has been constant up. The signal expected at the destination can be declared as: 
$Y_{d}[n]=Y_{s, d}[n]$

$$
\frac{S N R_{s, d}}{S N R_{s, r, d}}>10
$$

$Y_{d}[n]=Y_{s, d}[n]+Y_{s, r, d}[n]$

$0.1 \leq \frac{S N R_{s, d}}{S N R_{s, r, d}} \leq 10$

$Y_{d}[n]=Y_{s, r, d}[n]$

$$
\frac{S N R_{s, d}}{S N R_{s, r, d}}<0.1
$$

Where, SNRs, d and SNRs, r, d are the transmitter to destination channel and the transmitter -relay-destination channel SNR values correspondingly.

\section{III.Simulation Results and discussion}

The current simulation based study has been made for cooperative OFDM scheme in study with numerous parameters obtainable in Table 1.

Table 1: Summary of the simulated model parameters

\begin{tabular}{|l|l|}
\hline No. of bits used for text message & 1197 \\
\hline Channel Coding & $1 / 2$-rated Convolutional Encoder \\
\hline Modulation & QPSK \\
\hline OFDM Block size & 256 symbols \\
\hline Relaying Protocols & $\begin{array}{l}\text { Amplify and Forward(AAF) } \\
\text { and Decode and Forward(DAF) }\end{array}$ \\
\hline Signal Combining Scheme & $\begin{array}{l}\text { Equal ratio combining(ERC), } \\
\text { Fixed ratio(FRC), Signal to noise } \\
\text { ratio combing(SNRC) and } \\
\text { Enhanced Signal to noise ratio } \\
\text { combing (ESNRC) }\end{array}$ \\
\hline Channel & ITU Channels \\
\hline
\end{tabular}

In this part, simulation and theoretical results for the average BER are offered. All simulation results coincide to $\mathrm{N}=256$ and QPSK modulation. A block ITU Channels [9] was regarded. In Other hands, an autonomous channel implementation, was produced per transmitted OFDM-DWT signal. A two path uniform power delay outline was expected for the ITU Channels. $\sigma_{l}^{2}=1 / 2$. At the relay and destination stations, for decoding ideal channel state information and synchronization was expected. In Figure.1 theoretical and simulated BER versus SNR results for ideal relay decoding are shown. When the relay accurately forwards the transmitted source data, by using the destination MRC receiver. 


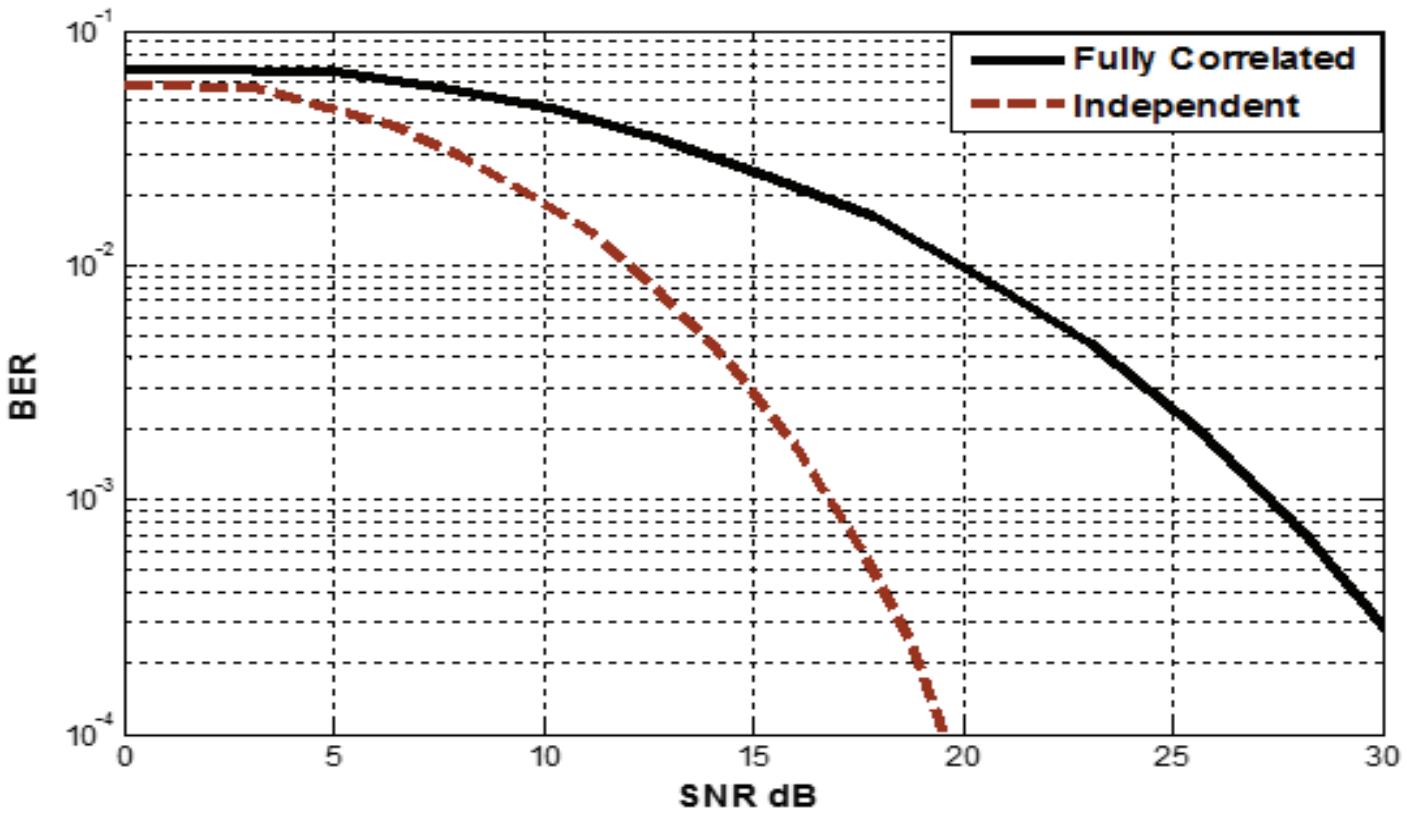

Figure. 1. Bit error presentation for ideal relay decoding.

Figure.2 displays the simulated end-to-end BER presentations with mapping diversity. The relay decoding is not ideal and it may or cannot retransmit accurate source data towards the destination

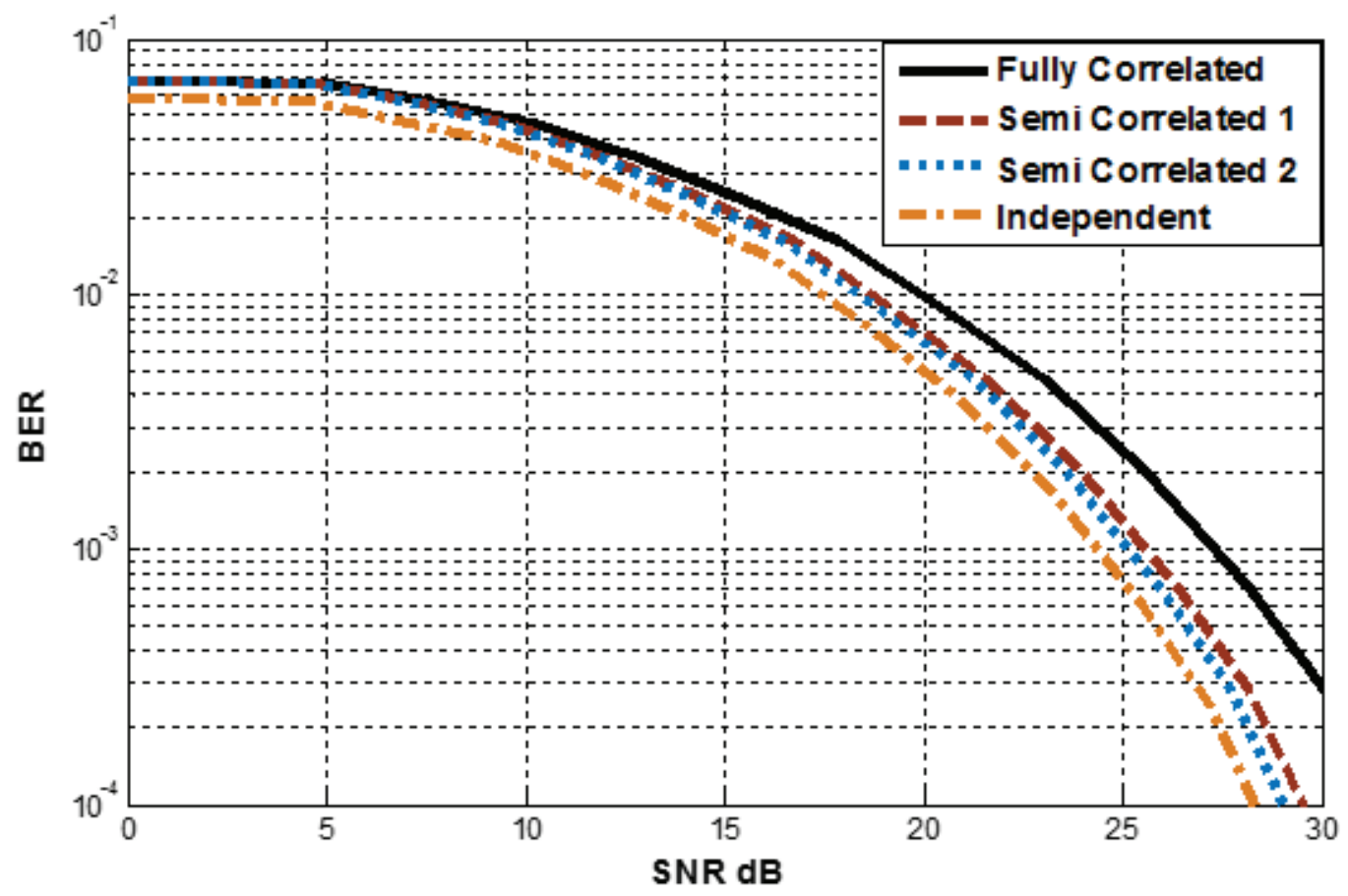

Figure. 2. Bit error performance for imperfect relay decoding. Semi Correlated 1 for $\Delta=2$, Semi Correlated 2 for $\Delta=4$

Figure. 3 displays the BER presentation of "completely correlated" and "independent" a situation against SNR at the destination for two not moving relay SNR values. These results show the influence of relay decoding errors on the end-to-end error presentation. 


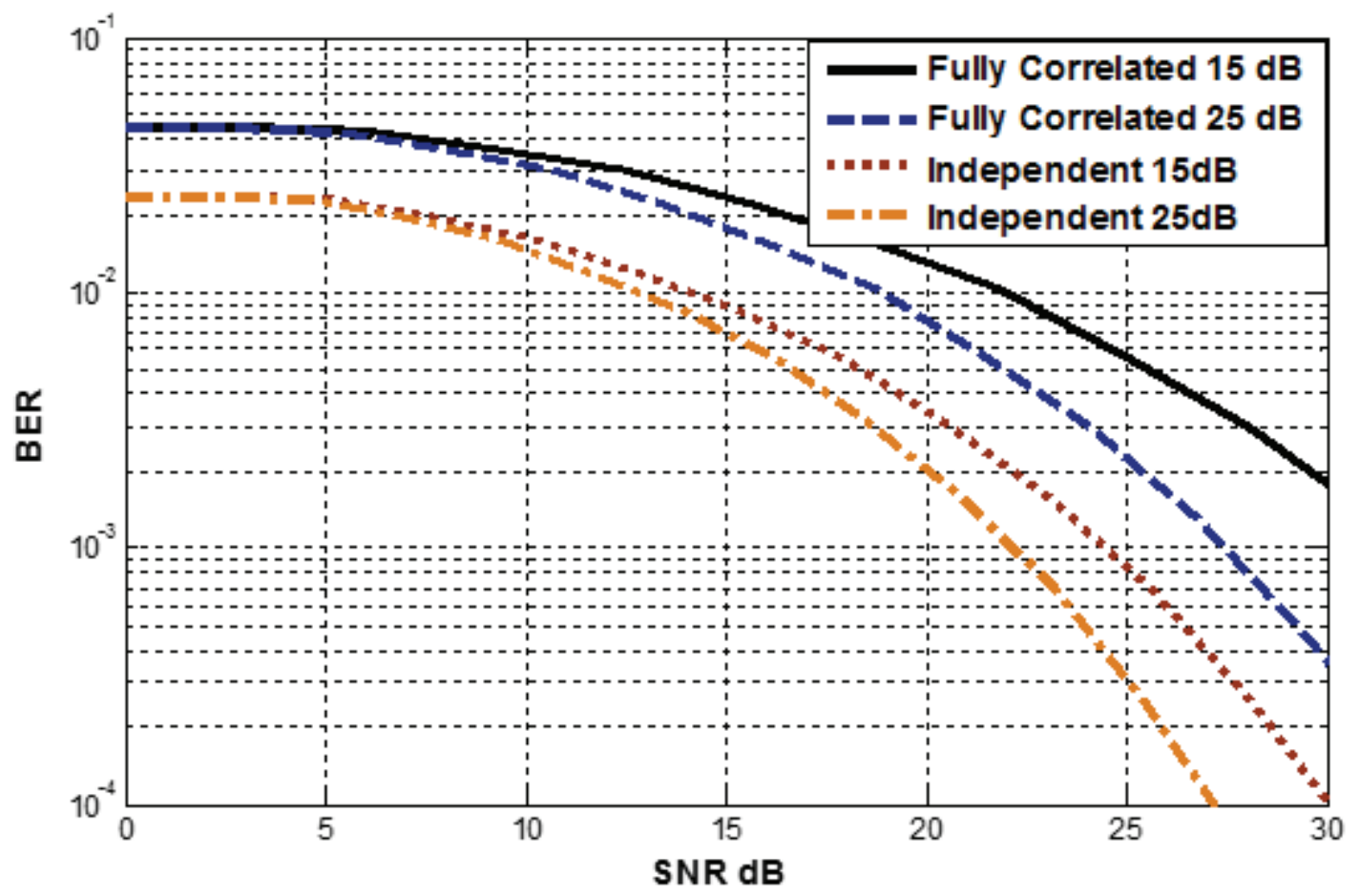

Figure. 3. Simulated BER for fixed relay SNR.

The results indicated in Figures. 2 and 3 explain the restriction of the simulated relay protocol .The presentation of this protocol can be enhanced, if the relay is permitted only to decode and retransmit, if the incoming signals are above an inevitable SNR threshold. This method minimize the probabilities of the incorrect transmission by the relay. The simulation results which more clarify the potential benefits of the diverse cooperative communication protocols when working with different combining methods. The transmission channel is expected to be an ITU Channels in which all frequency components are affected by the same fading coefficients. Figure 4 displays a plot of a four dissimilar combining methods. As can be understood from the plot on Figure 4, the SNRC and the ESNRC combining methods, which have an appreciated equal BER, have a much improved performance than the FRC and ERC combining methods. This might be assign to the detail that the SNRC and ESNRC methods need additional ITU Channels. Also, the FRC which has average information of the channel quality has improved performance than that of the ERC which has no information of the channel environments. A benefit of the AAF protocol is the low calculating power and bandwidth used in simply amplifying and forwarding the signal to the relay station without the essential for additional signal processing. 


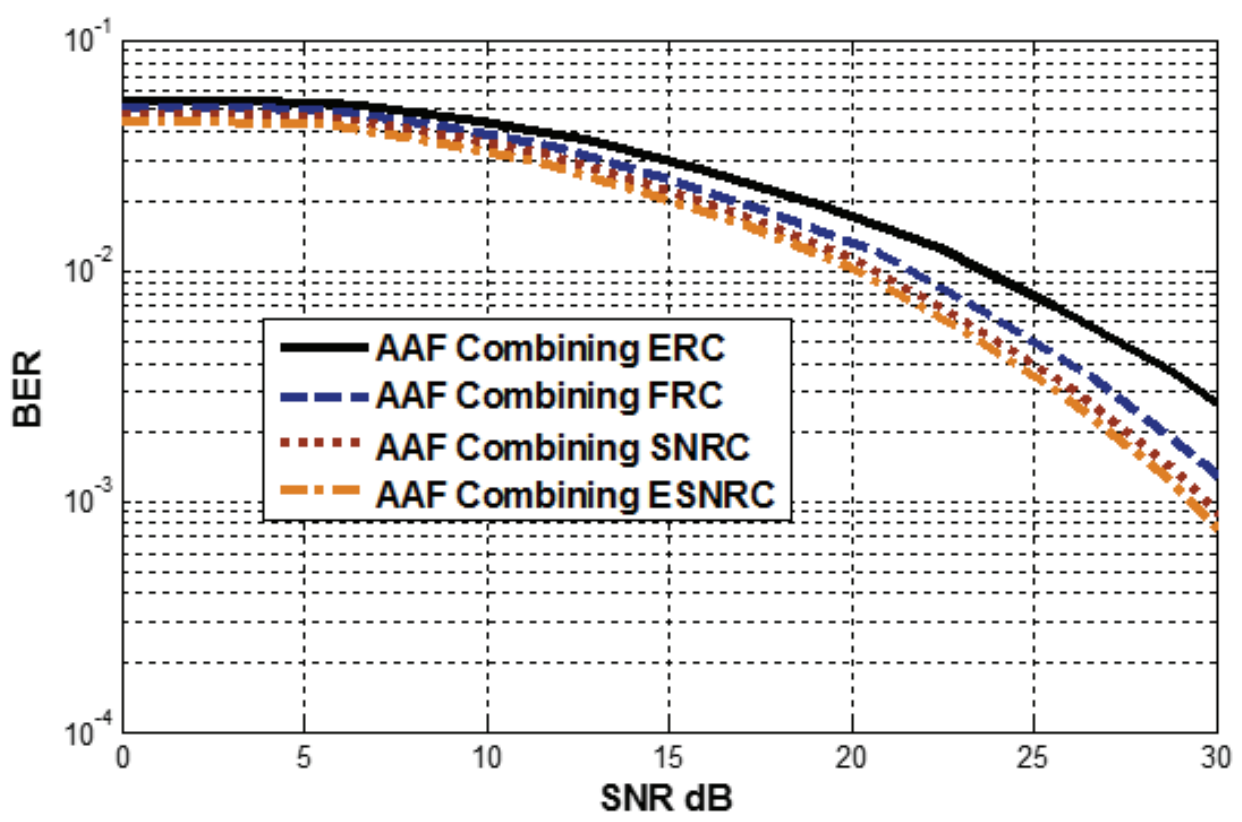

Figure 4: The different combining techniques are compared with each other with equidistant triangular placement of the source, the relay and the receiver for the AAF protocol. The best performance is obtained with the SNRC/ESNRC techniques.

The simulations are repetitive again but with the Decode and Forward protocol. Figure 5 shows the combining methods. The results specify poor performance for the combining approaches that don't need the exact channel situations. The ones which need average estimation or total estimation of the channel state achieve superior. Though, the greatest presentation is that of the ESNRC method. It is significant to note that the AAF protocol continuously has a well presentation with any combining way if the DAF protocol is realized without any error modifying appliance. The downside is that all, counting errors and noise, is amplified and transmitted along with the signal. Though, the MRC is the best combining way when an error correcting appliance is used.

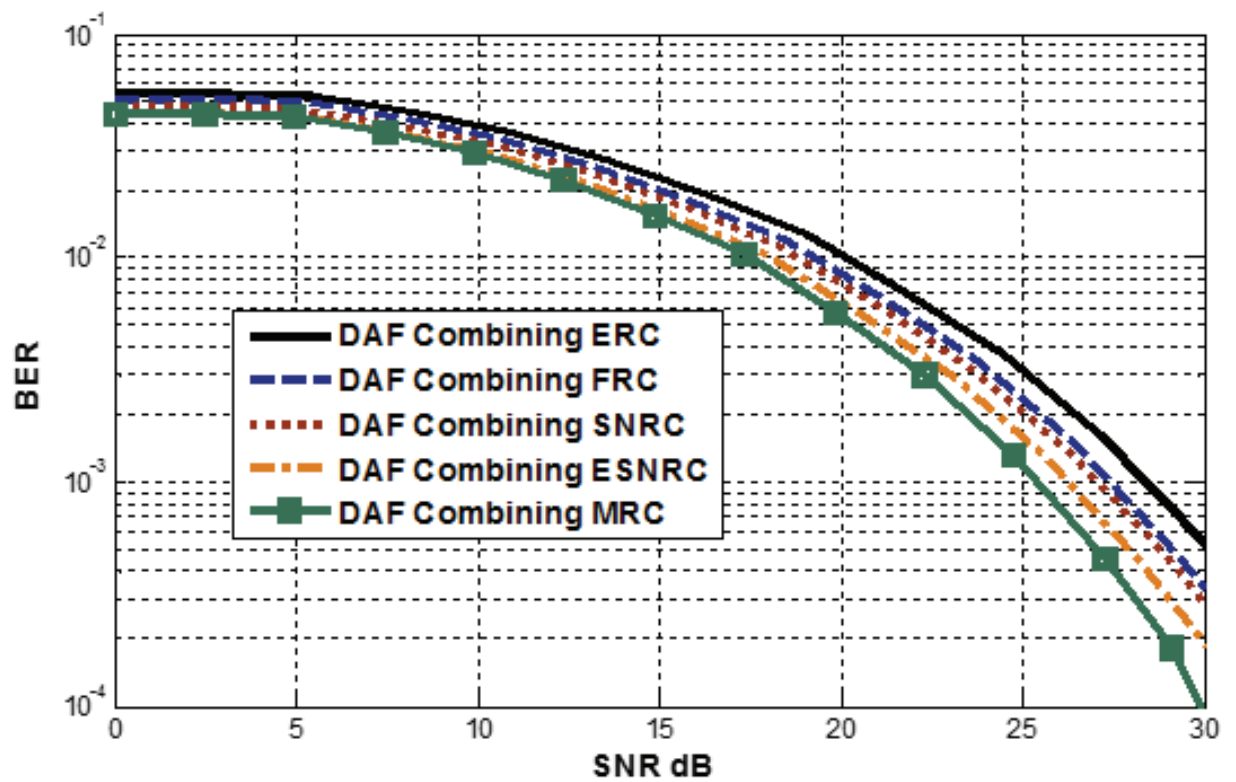

Figure 5: The different combining types are compared with each other with equidistant triangular placement of the source, the relay and the receiver for the DAF protocol. When an error correcting mechanism is implemented, the MRC has the best performance.

Figure 6 shows a presentation contrast of the AAF and DAF protocols. The result displays that the DAF protocol continuously results in a superior presentation than the AAF protocol when any combining method is 
used. This is because of the existence of amplified noise in the transmitted signal with best to the AAF protocol. Memory that this protocol is based on the amplification of the signal by the relay before being directed to the receiver. This has a negative result on the characteristic, of the signal expected at the destination because of the presence of noise in the amplified signal.

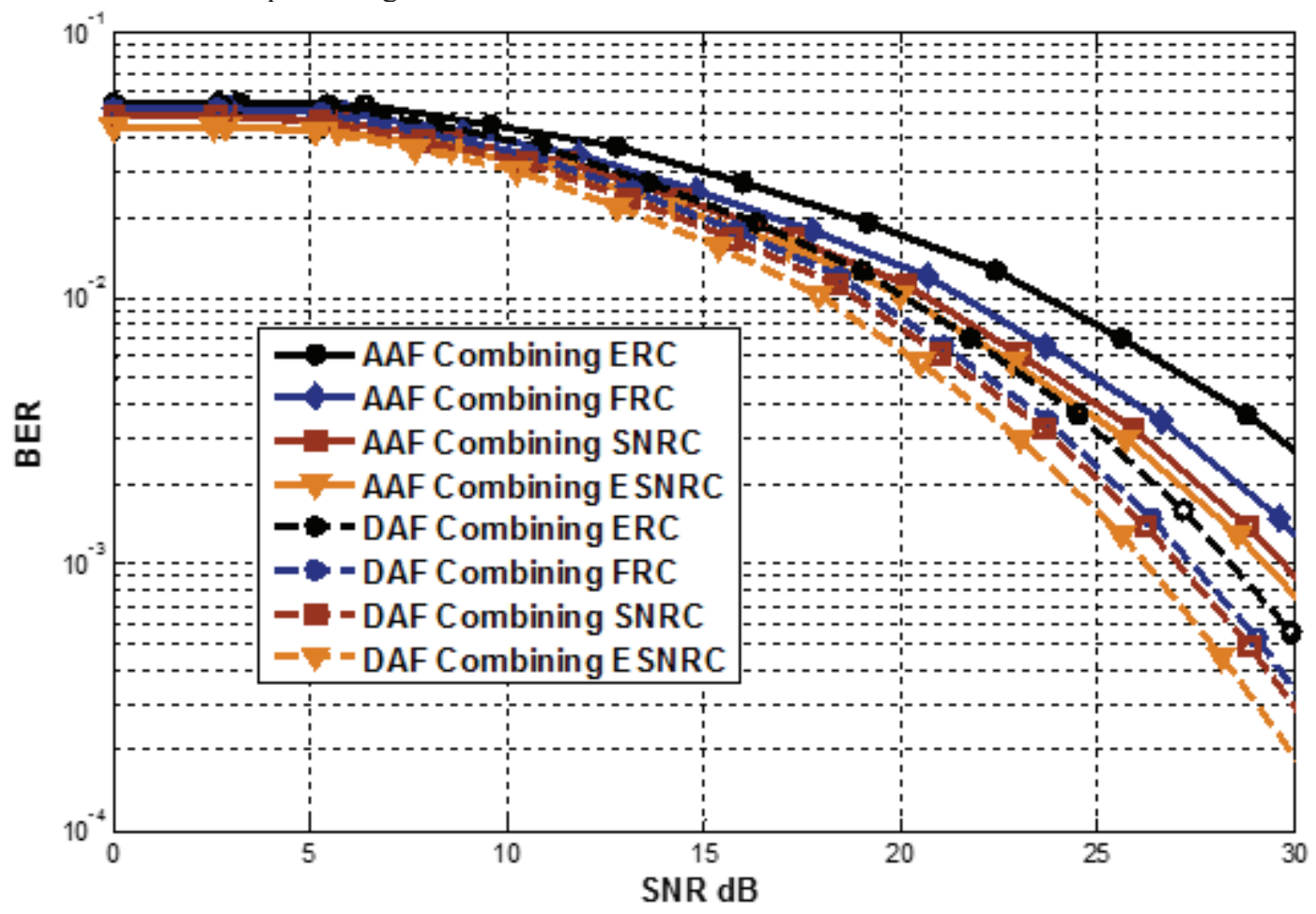

Figure 6: The two diversity protocols (AAF and DAF) are compared with each other. The DAF results in a better performance than the AAF for any combing technique employed.

The free space path loss of a wireless channel is associated to the square of the distance between the target and the base position. From Figures 7 and 8, it is displayed that any combining method is working with any protocol, well presentation is continuously common with the relay near to the transmitter. This is because the signal from the source reaches the relay without much reduction by the channel environments between the transmitter and the relay. This signal is too more processed at the relay and directed forwards to the destination. In the situation of the AAF protocol, both the noise and the signal are amplified at the relay which is rather beneficial as the signal with slight noise becomes amplified. This amplified signal is capable to resist the channel damages that might be bump as it is transmitted from the relay to the destination. In the situation of the combining approaches which need rough or whole estimation of the channel environments, it is suitable to classify the channel abilities earlier with the possible position of the relay. The ESNRC combing process which accomplished well in both AAF and DAF is used during to see the properties of variable relay positioning. Clearly in all situations where the relay is positioned closer to the destination, the presentation reductions. 


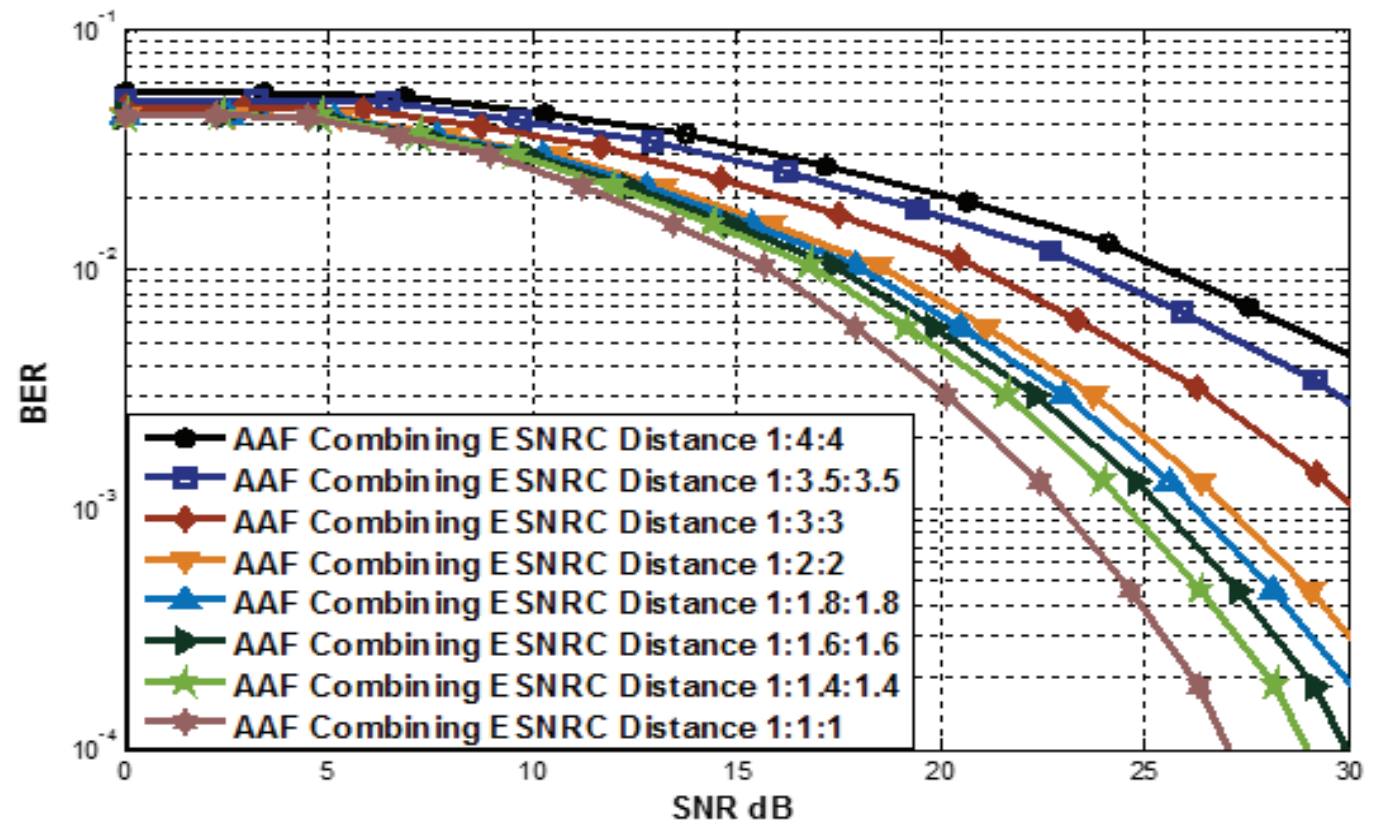

Figure 7: The effect of variable positioning is analyzed for the AAF protocol. Best performance is observed when the relay is exactly between the source and destination.

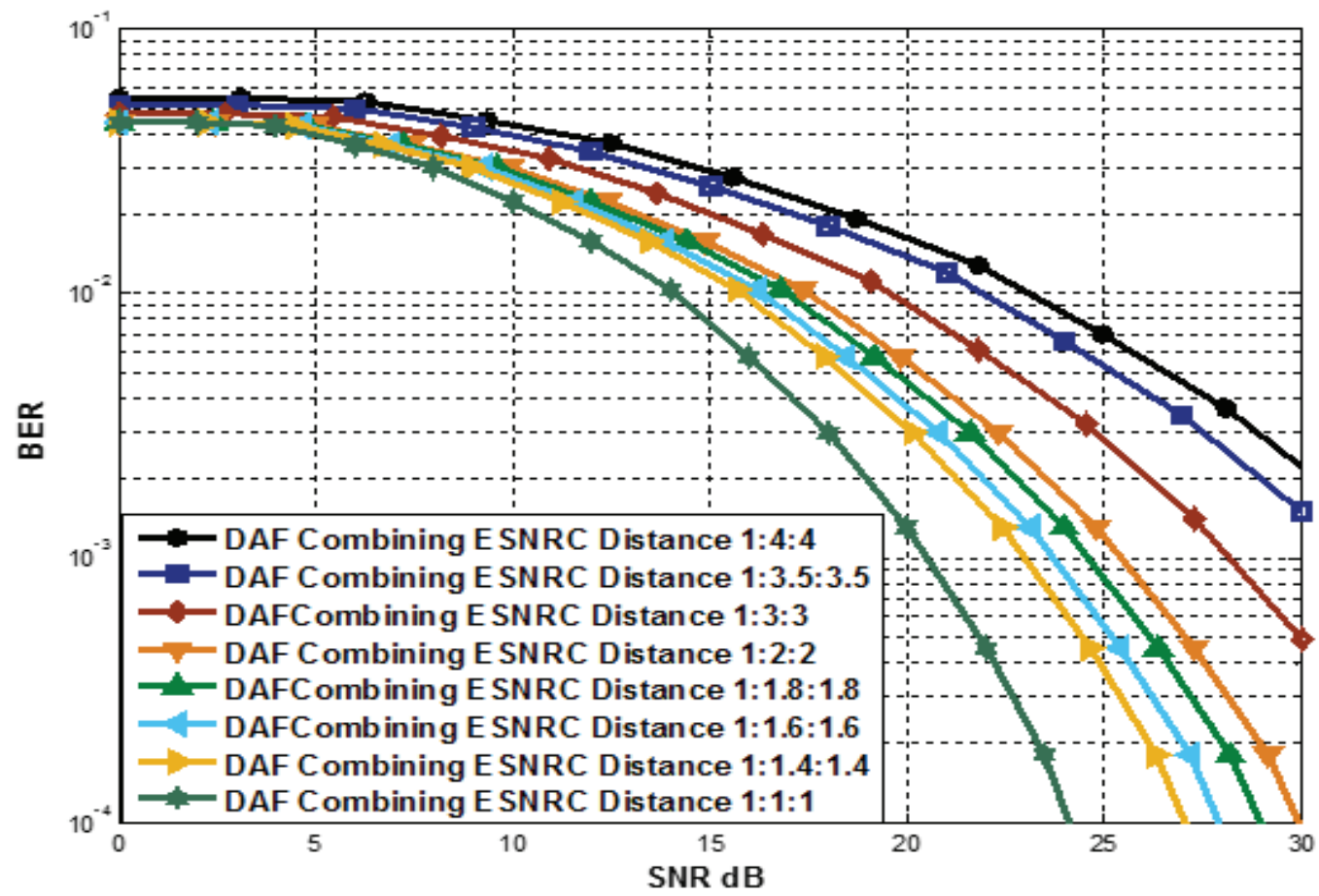

Figure 8: The effect of variable positioning is analyzed for the DAF protocol. Best performance is observed when the relay is exactly between the source and destination.

\section{Conclusions}

In this paper, we have suggested OFDM-DWT transmission method for collocated source relay communications. The relay re-maps the decoded changeable onto dissimilar subcarriers. We have analyzed the resulting theoretical BER presentation. Simulation results direct that the suggested system be able to advance the presentation of space diversity restricted relay communications. Also investigated the profits of cooperative 
diversity in wireless communication. Other conclusion from the simulation results is that the Amplify and Forward (AAF) protocol has its best performance when the Signal to Noise Ratio Combiner (SNRC) or the Enhanced Signal to Noise Ratio Combiner (ESNRC) method is working. The benefit of this protocol lies in the low calculating power and minimal bandwidth which it employs. Its disadvantage is that it amplifies and transmits all including noise. The Decode and Forward protocol though noted much improved performances than the Amplify and Forward protocol when any combination method is used. This is because of the existence of an error modifying code at the relay terminal and the detail that the noise is not amplified as in the situation of the AAF protocol. It has an optimal presentation when the Maximal Ratio Combiner (MRC) method is working. The drawback of the protocol is the great amount of calculating power it essential to work with. There was too the feature of relay locating and numerous conclusions were drawn with respect to the results got from the simulations. The DAF and AAF protocols working with the numerous combining methods have their greatest presentations when the relay is accurately between the source and destination. In variable the location of the relay regardless of the layout geometry, repeatedly low presentations were verified as the relay moved nearer to the destination. Presentation, though, improved as the relay was moved nearer to the signal source.

\section{References}

[1] A. H. M. Janani, T. Hunter, and A. Nosratinia, , "Coded cooperation in wireless communications: space-time transmission and iterative decoding," " Signal Processing, IEEE Transactions on., 2004.

[2] H. B. a. F. W. K. R.U.Nabar, "Fading relay channels: performance limits and space-Time signal design," IEEE Journal on Selected Areas in Communications., 2004.

[3] M. Yu and J. Li, " "Is amplify-and-forward practically better than decode-and-forward or vice versa"," Conf. ICASSP. , 2005.

[4] H. Y. a. E. D. C. B. Can, " "Hybrid forwarding scheme for cooperative relaying in OFDM based networks," " in Proc. IEEE ICC 2006, Istanbul, Turkey, June . 2006.

[5] M. Herdin, "“A chunk based OFDM Amplify-and-Forward relaying scheme for 4G mobile radio systems," in Proc. IEEE ICC 2006, Istanbul, Turkey, June., 2006.

[6] K. K. a. M. S. C. Hoymann, "“Multihop Communication in Relay Enhanced IEEE 802.16 Networks," " in Proc. IEEE Intl. Symp. Personal, Indoor and Mobile Radio Commun. (PIMRC 2006), Helsinki, Finland, Sept. , 2006.

[7] M. K. Lakshmanan and H. Nikookar, "A review of wavelets for digital wireless communication,"” Journal on Wireless Personal Communication, vol. 37, no.3-4, pp. 387-420,Springer, May., 2006.

[8] M. G. R. Dilmirghani, " "Wavelet Vs Fourier based UWB systems,"." 18th IEEE International Symposium on Personal, Indoor and Mobile Radio Communications, pp.1-5,Sep 2007.

[9] R. I.-R. M. International Telecommunication Union, "Guidelines for Evaluation of Radio Transmission Technologies for IMT-2000," 2007. 